\title{
Apoyo directivo y consecuencia de carrera en la conciliación trabajo-familia, en el sector de ventas al por menor (retail) en Chile
}

\author{
Andrés E. Jimenez-Figueroa ${ }^{1}$ y Miguel A. Bustamante ${ }^{2,3}$ * \\ (1) Facultad de Psicología, Universidad de Talca, Avda. Lircay s/n, Talca, Chile (e-mail: anjimenez@utalca.cl) \\ (2) Facultad de Economía y Negocios, Universidad de Talca, Avda. Lircay s/n, Talca, Chile. (e-mail: mabu@utalca.cl) \\ (3) Sistema de Posgrado de la Universidad Católica de Santiago de Guayaquil, Av. Carlos Julio Arosemena Km. 111/2 vía \\ Daule, Guayaquil, Ecuador.
}

* Autor a quien debe ser dirigida la correspondencia

Recibido Jun. 19, 2019; Aceptado Ago. 19, 2019; Versión final Sep. 4, 2019, Publicado Abr. 2020

\begin{abstract}
Resumen
El presente trabajo determina la relación entre apoyo directivo y consecuencia de carrera en la conciliación trabajo-familia, en el sector de venta al por menor en Chile. Se aplicó un cuestionario, previa certificación del comité de ética científica a una muestra aleatoria, estratificada y por redes, bajo el procedimiento de bola de nieve. Para el procesamiento de datos, se optó aplicar análisis de factores exploratorios mediante análisis paralelo robusto, por mínimos cuadrados no ponderados y rotación oblimin directo. Finalmente se modelaron los factores mediante análisis estructural confirmado por los índices de bondad de ajuste pertinentes. Entre los resultados, se determinaron dos factores de apoyo directivo y un factor de consecuencia de carrera que se confirmaron mediante cargas factoriales altas y significativas. Se concluye que los principios de la cultura trabajo-familia confirman la incidencia apoyo directivo sobre consecuencias de carrera y actúan reduciendo los efectos adversos sobre las consecuencias de carrera y, por lo tanto, reducen las eventuales tendencias de conflicto trabajo-familia.
\end{abstract}

\section{Directing support and career consequence in work-family conciliation, in the retail sector in Chile}

\begin{abstract}
The present work determines the relationship between managerial support and career consequence in workfamily conciliation, in the retail sector in Chile. A questionnaire was applied, after certification of the scientific ethics committee to a random sample, stratified, and by networks, under the snowball procedure. For the processing of data, it was decided to apply exploratory factor analysis through robust parallel analysis, by unweighted least squares and direct oblimin rotation. Finally, the factors were modeled by structural analysis confirmed by relevant goodness-of-fit indices. Among the results, two factors of managerial support and a factor of career consequence were determined and were confirmed by high and significant factorial loads. It is concluded that the principles of the work-family culture confirm the incidence of managerial support on career consequences and act reducing the adverse effects on the consequences of career and, therefore, reduce the possible tendencies of work-family conflict.
\end{abstract}




\section{INTRODUCCIÓN}

En general, la cultura trabajo-familia relaciona el trabajo y los roles familiares (Allen, 2001; Mauno, et al., 2005) y ha sido definida en términos de concepciones, creencias y valores respecto del grado en que la organización apoya y valora la integración del trabajo y la vida familiar (Thompson, et al., 1999), incrementa las oportunidades de los trabajadores (Mauno, et al., 2005) y fomenta políticas de bienestar en las organizaciones (Lewis y Smithson, 2001). Es por ello que, en la literatura es posible encontrar múltiples modelos de cultura trabajo-familia (Thompson, et al., 1999; Allen, 2001; Clark, 2001; McDonald, et al., 2005), que se han sintetizado en la relación de los trabajadores con los supervisores y que se manifiesta en el tiempo y la flexibilidad operacional (Casper et al., 2011). Por otra parte, Thompson, et al., (1999) sugiere que la cultura trabajo-familia se compone de tres componentes tales como demandas de tiempo, consecuencias en la carrera y apoyo directivo, al tiempo que, McDonald, et al., (2005) distinguen apoyo directivo, consecuencias en la carrera, expectativas de tiempo, percepciones de género y apoyo de los colaboradores. En este contexto, "consecuencia en la carrera" refiere a los efectos de las decisiones de apoyo directivo, que se evidencian en el apoyo brindado a la mujer por parte de la organización (Friedman y Greenhaus, 2001) y que le permite su desarrollo profesional; en tanto que, "consecuencia de la carrera" se relaciona con los efectos que el desempeño profesional tiene sobre la vida familiar (Machuca y Heredia, 2009) y que se observa en la postergación de la maternidad desplazando la dimensión familiar.

Desde la interacción trabajo-familia, Clark (2001) examinó los efectos de la flexibilidad temporal, operacional y el respaldo de los supervisores para asumir las responsabilidades familiares, coincidentemente, los estudios de Lapierre y Allen (2006) analizaron el respaldo de supervisores al uso de teletrabajo y al tiempo flexible que, en términos generales, establecen políticas de flexibilidad con la familia y los estilos de supervisión incidiendo tanto en la satisfacción personal como en la disminución de los conflictos de roles (Frye y Breaugh, 2004). Es por ello que, la presencia en las organizaciones de políticas trabajo-familia se asocian con altos niveles de desempeño organizacional, incrementos en la productividad y mejoramiento en la moral laboral (Frye y Breaugh, 2004) poniendo en evidencia variables significativas que Litchfield, et al., (2004) relacionan con lealtad y grado de identificación con la empresa, disminución de los índices de ausentismo, menor estrés e incremento en la productividad, entre otros resultados. Se aprecia, así mismo, que la percepción de ambientes de trabajo favorables incrementan el uso de beneficios y estimula actitudes positivas de satisfacción laboral y compromiso organizacional (Allen, 2001) que respaldan el balance familia - trabajo y reduce el conflicto en forma significativa (Thompson, et al., 1999).

Sobre la base de lo indicado en este trabajo se analizan dos de las dimensiones del modelo de Thompson, et al., (1999) por cuanto el apoyo directivo percibido, es precisamente el que incide sobre consecuencia de carrera (Beauregard y Henry, 2009; Shaffer et. al., 2011), permite comprender el equilibrio trabajo-familia, explicar el compromiso por el desempeño de las personas y eventualmente, potenciar los mecanismos que posibilitan una participación más equitativa de hombres y mujeres al mercado laboral (Allard, et al., 2011). En consecuencia, la gestión del equilibrio trabajo-familia proporciona a los empleados opciones que les permiten mejorar su calidad de vida y establecer una vinculación eficaz de la vida familiar con el trabajo (Casper, et al., 2011). La cultura trabajo-familia asume admás un rol importante en la definición de políticas de conciliación entre la organización y los supervisores (Thompson, et al.,1999), lo que es percibido y valorado positivamente por los empleados (Thompson et al., 2005), gestándose un equilibrio que se expresa en un correcto desempeño tanto en el trabajo como con en la familia, con mínimo conflicto de roles (Clark, 2001)

En general, los personales, independientes del género, aprecian elementos formales e informales así como tangibles e intangibles que vinculan trabajo y familia, como son la asignación de tareas, responsabilidades, medios de trabajo, cultura y clima organizacional (Thompson et al., 2005), sin embargo, el apoyo directivo resulta ser una dimensión vital de la gestión, puesto que las jefaturas, desde su rol de conductores, integran y unifican el desempeño y que como explica Allen (2001), evidencia el apoyo organizacional a la relación trabajo-familia consustanciales de la función directiva. En este mismo sentido, las propuestas metodológicas de McDonald, et al., (2005), incorporan dos dimensiones al modelo propuesto por Thompson et al., (1999), que se expresan en percepciones de género sobre la utilización de las políticas y el apoyo del compañero de trabajo, particularmente atractivas en empresas que potencian el desarrollo de carrera y políticas flexibles, en torno a sus trabajadores, sean estos, hombres o mujeres (Casper, et al., 2011). En suma y, sobre la base de lo señalado hasta este punto del análisis, las políticas trabajo-familia valoran componentes de inclusión que se expresan en términos del porcentaje de mujeres, el tamaño de la empresa, la competitividad del mercado laboral y la retención de los empleados valiosos (Machuca y Heredia, 2009), contribuyendo al desarrollo integral de la vida de los personales (Jansen, et al., 2010).

En síntesis, una política de trabajo-familia activa a favor las necesidades familiares (Allen, 2001; Mauno, et al., 2005), previene agotamientos, estrés, depresión, fatiga, entre otros (Jansen et al., 2010), eleva los niveles de satisfacción laboral y vital estimulando conductas corresponsables en el hogar (Dawley, et al., 2010; Kwak, 
et al., 2010), influye en relaciones de equidad e imparcialidad que deben ser reflexionadas en términos de conciliación trabajo-familia (Grandey y Cordeiro, 2002), mismas que este trabajo busca determinar en la relación que se establece entre la dimensión apoyo directivo sobre la dimensión consecuencia de carrera del sector al por menor en Chile.

\section{METODOLOGÍA}

Sobre la base del modelo teórico de Thompson, et al., (1999) que analizan la cultura trabajo-familia a partir de las dimensiones apoyo directivo y consecuencias, relación ratificada por McDonald, et al., (2005) quienes también analizan apoyo directivo como dimensión incidente sobre consecuencias en la carrera, el presente trabajo se plantea la hipótesis $\mathrm{H} 1$ : que las prácticas organizacionales de la dimensión apoyo directivo repercuten positivamente sobre la dimensión consecuencia de carrera, seguido del objetivo de este trabajo que busca establecer un modelo que determine la relación y dirección del efecto del apoyo directivo sobre consecuencia de carrera de los personales del sector al por menor, en Chile.

Es por ello que el presente trabajo se focaliza en la relación de las dimensiones apoyo directivo sobre consecuencia de carrera, teniendo en consideración que la dimensión consecuencia de carrera incluye reactivos, que se expresan en términos negativos, en consecuencia a fin de aislar efectos que enturbien los hallazgos, el presente trabajo se enfocó en estudiar, en forma exclusiva, si una gestión eficaz del apoyo directivo, produce el efecto de reducir los impactos adversos de consecuencia de carrera, como lo declara H1.A partir de $\mathrm{H} 1$ y el objetivo declarado, el presente trabajo es de carácter cuantitativo, transversal y exploratorio en la validación de reactivos y constructos (Hernández et al., 2016), además, es confirmatorio para validar factores de acuerdo con la teoría debidamente referenciada (Thompson, et al., 1996). Finalmente establece relaciones mediante modelamiento por ecuaciones estructurales, previo análisis factorial de las dimensiones. El tamaño de la muestra se obtuvo a través de un muestreo aleatorio, estratificado por grupos con un sub muestreo causal y por redes, bajo el procedimiento de Bola de Nieve (Hernández et al., 2016).

Previo a la aplicación del instrumento, la comisión de ética de investigación de la Universidad de Talca, revisó y aprobó la realización del instrumento bajo las normas de ética, optándose por realizar el cuestionario en forma anónima bajo el principio de consentimiento informado. El cuestionario fue aplicado en modalidad online, previa explicación de las bases y los términos de anonimato y confidencialidad. Se alcanzó una duración promedio de respuesta de aproximadamente de 40 minutos.

\section{Descripción de los instrumentos}

El Cuestionario de Cultura Trabajo-Familia fue elaborado por Thompson, et al., (1996), consta de 20 ítems y su formato de respuesta es una escala Likert de 7 puntos donde el 1 representa totalmente en acuerdo y el 7 representa totalmente en desacuerdo. En sus detalles, el cuestionario mide tres componentes de la cultura trabajo-familia: Apoyo Directivo, Consecuencias de Carrera, y Demandas de Tiempo. La confiabilidad general alcanza un índice de 0,86, en tanto que, ha sido de 0,85 para Apoyo Directivo (11 ítems), de 0,79 para Consecuencias de Carrera (5 ítems) y de 0,76 para Demandas de Tiempo (4 ítems). Sin embargo, dada la naturaleza semántica de los reactivos, la hipótesis y objetivos declarados, se analiza en forma exclusiva en este trabajo la incidencia de apoyo directivo sobre consecuencia de carrera.

\section{Análisis Factorial Exploratorio y confirmatorio}

Se siguieron las fases recomendadas por Freiberg et al., (2013), se optó por aplicar análisis paralelo (PA) mediante la matriz de dispersión de variables policóricas, análisis robusto de los mínimos cuadrados no ponderados (RULS) y rotación oblimin directo, puesto que permite asumir relaciones de interdependencia entre ítems cualitativos y factores latentes que pueden resultar correlacionados (Freiberg et al., 2013).

Sobre la base de los constructos analizados, se midieron las percepciones de los entrevistados sobre los reactivos mediante escalas policóricas de 7 puntos (Freiberg et al., 2013), lo que hace posible asumir se superan los supuestos de no normalidad de las escalas además de poder relacionar variables latentes que pudiesen pasar inadvertidas si se aplicara rotación ortogonal. Así mismo, siguiendo las orientaciones de Freiberg et al., (2013), se optó por comunalidades superiores $(\geq 0,4)$, asegurando correlaciones directas y fuertes entre ítems y constructos. Además se exigieron cargas factoriales altas $(\geq 0,4)$ para excluir reactivos con baja correlación. Finalmente, se evalúo la confiabilidad de cada uno de los constructos mediante alfa de Cronbach y Omega de McDonald (Freiberg et al., 2013), además del índice de error raíz cuadrático medio, RMSR y se verificó el número de ítems mínimo $(\geq 3)$ para cada constructo. Se consideró así mismo la medida de adecuación KMO ( $\geq 0,80$; Kaiser, 1970), los coeficientes de significancia de Bartlett y se analizó la varianza explicada (Freiberg et al., 2013) para obtener configuraciones simples e interpretables. 


\section{Modelamiento Estructural (SEM)}

Ejecutadas las recomendaciones de Baño y Kaplan (Freiberg et al., 2013), se verificó la existencia de casos atípicos con valores extremos o poco significativos para asegurar que los resultados e interpretación de las estimaciones multivariantes fuesen pertinentes. Para confirmar ítems, se definieron las variables como policóricas para ser analizadas mediante el programa Factor aplicando análisis factorial exploratorio (AFE). Luego se modelaron las variables latentes mediante ecuaciones estructurales. Los análisis de bondad de ajuste se realizaron considerando los índices absolutos Estadístico-Radio de Verosimilitud de Chi-Cuadrado (CMIN/DF $\leq 3$ ), el Índice GFI $(\geq 0,9)$ que mide la variabilidad explicada por el modelo, el error de aproximación cuadrático medio RMSEA $(\leq 0,05)$ y el índice de error cuadrático medio RMR $(\approx 0)$. Además se verificaron las medidas de ajuste Comparative Fit Index (CFI $\geq 0,9)$, Goodness of Fit Index (GFI $\geq 0,9$ ), incremental Adjusted Goodness of Fit Index (AGFI $\geq 0,8$ ), Goodness of Fit Index without diagonal values (GFI $\geq 0,8$ ) (Freiberg et al., 2013). El proceso de datos se realizó a través de los programas SPSS v.21, Factor v.10.5.03 y AMOS v.21 (Build 1178) para Windows.

\section{RESULTADOS}

A continuación, se detallan los resultados de la investigación comenzando con una breve síntesis del sector al por menor en chile. Luego se sigue con el análisis descriptivo de la muestra, el análisis de género, el análisis factorial exploratorio, el análisis de estimadores y finalmente el modelamiento estructural

\section{El sector al por menor en Chile}

En 1975 se inaugura el primer Jumbo y a comienzos de los 80' se inicia incipientemente el boom de los centros comerciales con la creación del centros comerciales como Apumanque y Parque Arauco, tendencia que se extiende a regiones a partir de los años 90', dando pie a una industria que en Chile ha mostrado un gran dinamismo durante los últimos años (Fundación sol, 2008). Ya en el año 2006 las ventas del sector al por menor crecieron un 6,9\% respecto al año anterior alcanzando un total de US\$31 mil millones aproximadamente, representando un $22 \%$ del PIB y a la fecha se extiende hacia otros países de la región tales como Argentina, Perú y Colombia.

Desde la perspectiva del empleo, representa sobre el $50 \%$ a nivel mundial y $68 \%$ para Chile y su peso en el PIB alcanza el $66 \%$ a nivel global y $64 \%$ para Chile, evidenciando dinámicas laborales y organizacionales innovadoras (Edgell, et al., 2016). Así mismo, dado su peso relativo en términos de su peso económico, oferta masiva de empleo flexible y baja cualificación relativa dentro del sector servicios, ha sido objeto de diversas investigaciones empíricas y reflexión teórica a nivel global (Bank, et al., 2018; Tilly y Carre, 2011; Warhurst y Nickson, 2007). En consecuencia, se trata de un sector, el sector al por menor profusamente analizado que se constituye en sí misma en una de las mayores fuentes laborales en nuestro país y uno de los sectores con mayor riesgo psicolaboral viéndose, por ende, afectada la calidad de vida de quienes allí laboran y cuyos efectos adversos se manifiestan en rotación y ausentismo provocando grandes pérdidas económicas en término de costo de desvinculación y de capacitación de nuevos empleados (Fundación sol, 2008).

\section{Análisis descriptivo de la muestra}

El instrumento se aplicó a 618 personas de la población objetivo constituida por trabajadores que se desempeñan en el sector al por menor en 14 de las 15 regiones de Chile y que contestaron correctamente la batería de reactivos del instrumentos. De los 618 contactos, 441 son mujeres $(71,4 \%)$ y 117 son hombres $(28,6 \%)$. Sus edades se encuentran en un rango de 21 a 65 años, cuyo promedio general es de 38 . La mayoría de los trabajadores $(93,5 \%)$ tiene un contrato de trabajo indefinido y con jornada completa $(95,2 \%)$. Se observa en el nivel de estudios de los trabajadores, que la mayor parte presenta educación universitaria completa $(42,6 \%)$ seguida de educación media incompleta $(29,2 \%)$. En el estado civil, prevalecen los trabajadores casados (36,7\%), viviendo en pareja un $17,6 \%$ y separado un $6,6 \%$. En general, el $66,1 \%$ señala ser el principal proveedor de su respectivo hogar. Adicionalmente, un $25,4 \%$ de los empelados señalan percibir ingresos entre $\$ 975.001$ - $\$ 1.575 .000$ (US\% 1.393 - US $\$ 2.250 .000$ ) seguido de un $21,2 \%$ que se ubica en el rango de $\$ 300.001$ y $\$ 450.000$ (US\$ 429 - US\% 643). Por último, respecto a las responsabilidades con el hogar, el $14,7 \%$ señala estar a cargo de un adulto mayor y el $69,7 \%$ tiene hijos a los que cuidar, en tanto que, para ayudarse, tan solo el 12,5\% cuenta con servicio doméstico a tiempo completo o parcial.

\section{Análisis de género}

Desde la perspectiva de género, las personas independiente de que sean hombres o mujeres, declaran que lo más importante es el grupo familiar en sus vidas, presentando algunas diferencias en términos de intereses y energías dedicadas al trabajo o a la familia, confirmando el hecho que familia y trabajo se afectan 
mutuamente (Thompson, et al., 1999) y que en general se valoran las prácticas laborales positivas como la flexibilidad en el horario y beneficios organizacionales tales como bonos, comisiones, metas, entre otras, que tienden a enriquecer la vida familiar.

Por el contrario, se aprecia que el estrés laboral intensifica el conflicto trabajo-familia (Casper, et al., 2011) y que, desde la perspectiva de género, la intensidad en que hombres o mujeres perciben por una parte el estrés y por otra el bienestar subjetivo (Spielberg, et al., 2000) se traducen en el punto de partida para potenciar la familia en el contexto empresarial, lo cual puede favorecer el equilibrio trabajo-familia analizado en este trabajo (Debeljuh y Jáuregui, 2004). Sin embargo, de los datos analizados por sexo, no se observan, en general, diferencias significativas en las apreciaciones de hombre o mujeres, a excepción de la variable "demandas de tiempo organización" que efectivamente presenta diferencias de género significativas (Sig. = 0,015).

\section{Análisis factorial exploratorio}

Como se detalla en la Tabla 1, el factor 1 de apoyo directivo concentra cinco (5) reactivos (V14, V15, V16, V18 y V20) correlacionados en rangos altos $(\geq 0,55 \mathrm{y} \leq 0,71)$ que se expresa en cargas factoriales superiores alcanzando una varianza explicada de $44 \%{ }^{*}$ significativa.

Tabla 1: Análisis factorial exploratorio de los constructos apoyo directivo- consecuencias de carrera

\begin{tabular}{|c|c|c|c|c|c|c|c|}
\hline \multicolumn{3}{|c|}{ Apoyo Directivo } & & \multicolumn{3}{|c|}{ Consecuencia De Carrera } & \multirow{2}{*}{$\begin{array}{l}\text { Items } \\
\text { Excluidos }\end{array}$} \\
\hline Variable & Factor 1 & Factor 2 & Comunalidad & Variable & Factor 1 & Comunalidad & \\
\hline V 1 & & 0,642 & 0,467 & & & 0,045 & $\vee 7$ \\
\hline$\vee 2$ & & 0,868 & 0,733 & & & 0,129 & V 9 \\
\hline V 3 & & 0,631 & 0,392 & V 12 & 0,404 & 0,163 & \\
\hline V 5 & & 0,755 & 0,582 & V 13 & 0,420 & 0,177 & \\
\hline$\vee 8$ & & 0,708 & 0,496 & V 19 & 0,823 & 0,678 & \\
\hline V 14 & 0,711 & & 0,497 & & & & \\
\hline V 15 & 0,610 & & 0,353 & & & & \\
\hline V 16 & 0,570 & & 0,303 & & & & \\
\hline V 17 & & & 0,130 & & & & $\vee 17$ \\
\hline V 18 & 0,550 & & 0,271 & & & & \\
\hline V 20 & 0,679 & & 0,565 & & & & \\
\hline $\begin{array}{l}\text { Varianza } \\
\text { Expolicada }\end{array}$ & $44,0^{*}$ & $19,7^{*}$ & & & $52,5^{*}$ & & \\
\hline \multicolumn{3}{|c|}{ Kaiser-Meyer-Olkin (KMO) } & 0,86006 & & & 0,60457 & \\
\hline \multicolumn{3}{|c|}{ Standardized Cronbach's alpha } & 0,813542 & & & 0,539632 & \\
\hline \multicolumn{3}{|c|}{ McDonald's Omega } & 0,806978 & & & 0,560207 & \\
\hline \multicolumn{3}{|c|}{$\begin{array}{l}\text { Root Mean Square Error of } \\
\text { Approximation (RMSEA) }\end{array}$} & 0,084 & & & 0,347 & \\
\hline \multicolumn{3}{|c|}{ Comparative Fit Index (CFI) } & 0,957 & & & 0,141 & \\
\hline \multicolumn{3}{|c|}{ Goodness of Fit Index (GFI) } & 0,995 & & & 0,967 & \\
\hline \multicolumn{3}{|c|}{ Adjusted Goodness of Fit Index (AGFI) } & 0,992 & & & 0,933 & \\
\hline \multicolumn{3}{|c|}{$\begin{array}{l}\text { Adjusted Goodness of Fit Index without } \\
\text { diagonal values(AGFI) }\end{array}$} & 0,984 & & & 0,627 & \\
\hline
\end{tabular}

El factor 2 por su parte, integra también cinco (5) variables (V1, V2, V3, V5, V8) con cargas factoriales altas $(\geq 0,65 y \leq 0,75)$ mostrando una varianza explicada de $19,7 \% *$ significativa. Se aprecia sin embargo, que de los reactivos que componen la dimensión apoyo directivo, resulta excluido el ítem V17 el cual muestra un índice de comunalidad muy bajo $(0,130)$. Por su parte, la dimensión consecuencia de carrera concentra los reactivos $(\mathrm{V} 12, \mathrm{~V} 13, \mathrm{~V} 19)$ en un solo factor relevante con cargas factoriales relativamente altas $((\geq 0,40 \mathrm{y} \leq$ 0,82 ) alcanzando una varianza explicada significativa de $52,5 \%{ }^{*}$. Se excluyen en este caso las variables $\mathrm{V} 7$ y V9, puesto que éstos muestran índices de comunalidad muy bajos $(0,045$ y 0,129$)$. 


\section{Análisis estructural}

Sobre la base del análisis factorial previamente realizado, se procedió a procesar los datos mediante análisis estructural considerando los procedimientos de covarianzas y varianzas como se muestra en la figura 1. La relación inversa que se observa en la Figura 1 entre los factores de apoyo directivo (FAD1 y FAD2) sobre la dimensión de consecuencia de carrera (FCC1) es la resultante del sentido de redacción de los reactivos de esta dimensión del instrumento, los cuales, en todos los casos incluidos en el análisis (V12, V13 y V19), se expresan en términos negativos, en consecuencia, la gestión eficaz del apoyo directivo, produce el efecto de reducir el impacto de adverso de éstos sobre la persona.

En general se aprecia que los indicadores de covarianza y de varianza muestran índices idénticos para la

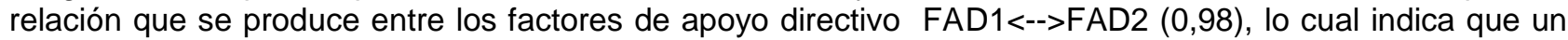
incremento en un punto de uno de los factores incide en 0,98 puntos sobre el otro. Por otra parte, las relaciones de covarianza y varianza que se presentan entre FAD1 <-->FCC1 son inversos y cercanamente similares $\left(-0,45^{* * *}\right.$ y $\left.-0,41\right)$ entre apoyo directivo y consecuencia de carrera, en consecuencia un incremento en un punto en uno de los factores incide reduciendo en $0,45 \circ 0,51$ el factor relacionado.
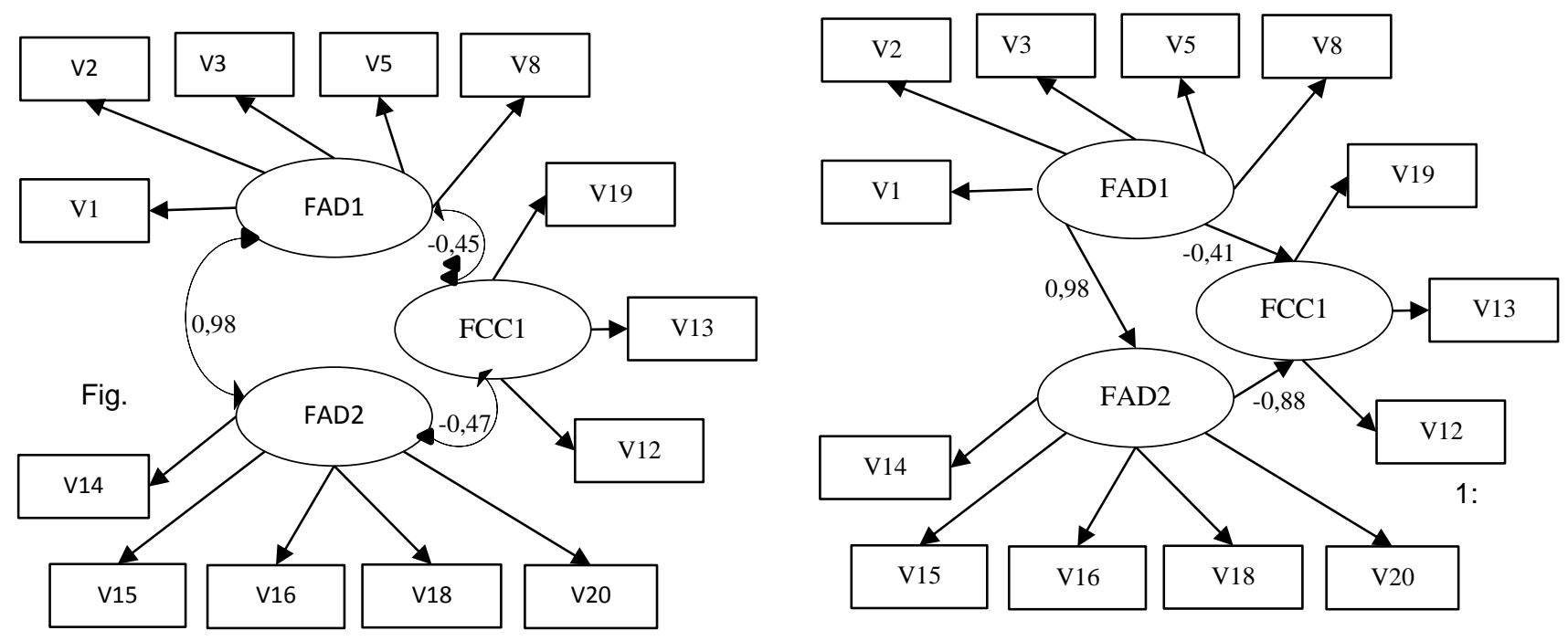

Fig. 1: Modelado confirmatorio mediante covarianzas y varianzas

Por último en esta fase general de análisis se aprecia que las relaciones de covarianza y varianza entre los factores de apoyo directivo y consecuencia de carrera FAD2<-->FCC1 es también inversa pero alcanzando índices relativamente diferentes en sus efectos $\left(-0,47^{* * *}\right.$ y $\left.-0,88\right)$, siendo más intensa la relación de varianza entre estos factores.

\section{Análisis de estimadores}

La tabla 1 muestra los estimadores y valores estándar del análisis estructural de las dimensiones de apoyo directivo y consecuencia de carrera. Se observa que los estimadores de los reactivos se expresan en valores positivos alrededor de 1 y con valores estándares ubicados perfectamente en el rango esperado $(0 \geq$ estándar $\leq 1$ ) y significativos al $1 \%\left({ }^{* * *}\right)$, en consecuencia, se puede afirmar que el ajuste realizado es correcto y los valores estimados representan adecuadamente la realidad. Por otra parte, los estimadores de los factores (FAD1, FAD2 y FCC1) alcanzan índices correctos alrededor de 1 con valores estandarizados correctos $(0$ y 1$)$, significativos al $1 \%\left(^{* * *}\right)$ a excepción de FAD2 que resulta no significativo $(0,216)$.

\section{Modelamiento estructural}

La tabla 3 presenta los índices del modelamiento estructural entre los factores de apoyo directivo y consecuencia de carrera. En general se aprecia que los estimadores del análisis de covarianza resultan adecuados y los valores estándar se encuentran en el rango esperado ( 0 a 1$)$. Por otra parte, los índices de varianza son adecuados y se ubican en el rango pertinente $(0$ a 1$)$ aunque no resultan todos significativos. Se aprecia que las relaciones de varianza entre los factores FAD1<-->FAD2, es positiva $\left(0,983^{\star \star *}\right)$ y significativa al $1 \%$ en tanto que entre $\mathrm{FCC} 1<-->\mathrm{FAD} 1$ es positiva $(0,414)$ pero no significativa. Por último, la relación entre FCC1<-->FAD2 resulta inversa $(-0,881)$ y no significativa. 
Tabla 2: Estimadores y valores estándar

\begin{tabular}{|c|c|c|c|c|c|c|c|c|c|c|}
\hline \multicolumn{6}{|c|}{ Análisis de Covarianzas } & \multicolumn{5}{|c|}{ Análisis de Varianzas } \\
\hline & Estimador & S.E. & C.R. & $P$ & Estándar & Estimador & S.E. & $C . R$. & $P$ & Estándar \\
\hline FAP1 & 1,222 & 0,118 & 10,336 & $* * *$ & & 1,222 & 0,118 & 10,336 & $* * *$ & \\
\hline FAP2 & 1,557 & 0,143 & 10,887 & *** & & 0,053 & 0,043 & 1,238 & 0,216 & 0,966 \\
\hline FCC1 & 1,236 & 0,139 & 8,901 & *** & & 0,950 & 0,125 & 7,612 & $* * *$ & 0,231 \\
\hline $\mathrm{V} 1$ & 1,017 & 0,067 & 15,270 & $* * *$ & 0,297 & 1,017 & 0,067 & 15,270 & $* * *$ & 0,546 \\
\hline V2 & 0,867 & 0,062 & 13,943 & *** & 0,458 & 0,867 & 0,062 & 13,943 & *** & 0,649 \\
\hline V3 & 1,150 & 0,069 & 16,572 & $* * *$ & 0,614 & 1,150 & 0,069 & 16,572 & $* * *$ & 0,310 \\
\hline V5 & 1,394 & 0,085 & 16,442 & *** & 0,284 & 1,394 & 0,085 & 16,442 & *** & 0,375 \\
\hline V8 & 0,770 & 0,056 & 13,696 & *** & 0,344 & 0,770 & 0,056 & 13,696 & $* * *$ & 0,664 \\
\hline V20 & 1,066 & 0,074 & 14,348 & *** & 0,605 & 1,066 & 0,074 & 14,348 & $* * *$ & 0,594 \\
\hline V18 & 0,872 & 0,059 & 14,901 & $* * *$ & 0,468 & 0,872 & 0,059 & 14,901 & $* * *$ & 0,468 \\
\hline V16 & 0,871 & 0,061 & 14,174 & $* * *$ & 0,594 & 0,871 & 0,061 & 14,174 & $* * *$ & 0,605 \\
\hline V15 & 1,474 & 0,090 & 16,411 & $* * *$ & 0,664 & 1,474 & 0,090 & 16,411 & $\star \star \star *$ & 0,344 \\
\hline V14 & 2,109 & 0,126 & 16,705 & *** & 0,375 & 2,109 & 0,126 & 16,705 & $* * *$ & 0,284 \\
\hline V12 & 0,777 & 0,100 & 7,752 & $* * *$ & 0,310 & 0,777 & 0,100 & 7,752 & $* \star *$ & 0,614 \\
\hline V13 & 1,071 & 0,091 & 11,750 & $* * *$ & 0,649 & 1,071 & 0,091 & 11,750 & $* * *$ & 0,458 \\
\hline V19 & 1,357 & 0,091 & 14,962 & $* * *$ & 0,546 & 1,357 & 0,091 & 14,962 & $* \star *$ & 0,297 \\
\hline
\end{tabular}

Tabla 3: índices de modelamiento estructural

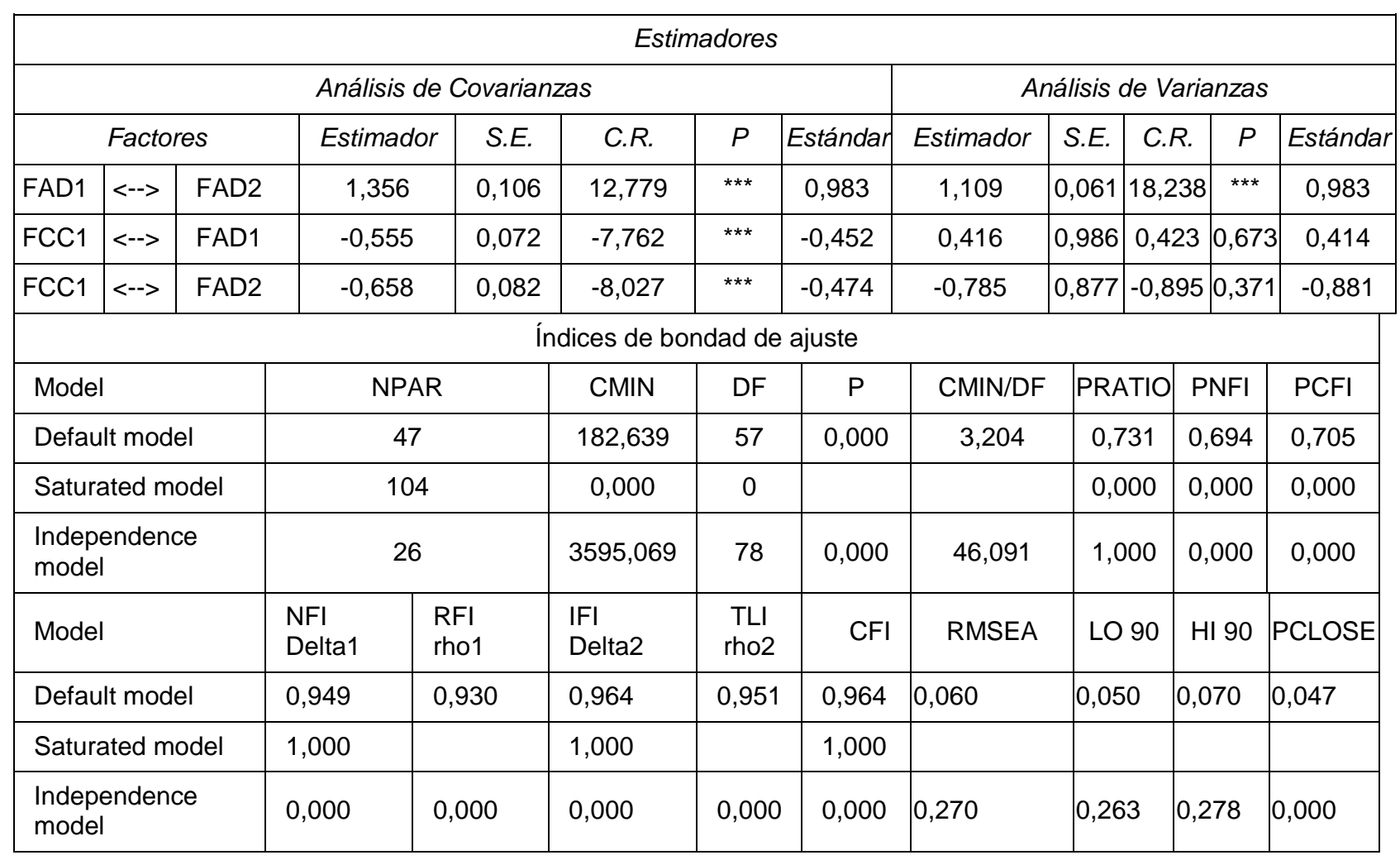

Finalmente se aprecia en la tabla 3 que los índices de bondad de ajuste confirman progresivamente el análisis realizado. Se obtiene un índice CMIN/DF de 3,204 ( $\leq 5)$ ubicado en el rango adecuado. Se observan además índices de modelamiento (NFI, TLI, CFI) todos altos $(\geq 0,9)$. Finalmente el índice de error RMSEA es de 0,06 suficientemente bajo $(\leq 0,08)$ para ratificar el ajuste logrado. 


\section{DISCUSIÓN}

Los dos factores de apoyo directivo que integran cinco componentes cada uno se determinan altamente correlacionados (en términos de covarianza y varianza 0,98 ) ratificando el vínculo ya previsto en la teoría (Allen, 2001; Mauno, et al., 2005), además, ambos inciden sobre el factor único de consecuencia de carrera (Jansen et al., 2010) con tres reactivos (V12, V13, V19). Seguidamente, el apoyo directivo percibido en este trabajo efectivamente incide de forma inversa, en términos de covarianza $-0,45^{\star * *},-0,47^{\star * *}$ en forma significativa y en términos de varianza $-0,41,-0,88$ y en forma no significativa sobre consecuencia de carrera (Beauregard y Henry, 2009; Shaffer et. al., 2011) lo que permite inferir que una gestión eficaz del apoyo directivo reduce los efectos adversos sobre consecuencia de carrera. Adicionalmente, que la incidencia inversa de los factores de apoyo directivo sobre consecuencias de carrera, confirma los hallazgos de estudios de conciliación trabajo-familia (Machuca y Heredia, 2009) ratificando los hallazgos de McDonald, et al., (2005) que relaciona también apoyo directivo con consecuencias en la carrera, en tanto genera beneficios que impactan tanto a los trabajadores como a las propias empresas, por una parte, incrementando la capacidad de las organizaciones para enfrentar cambios, potenciar la productividad y efectividad con mínimo ausentismo y rotación de personales (Casper et al., 2011), como se aprecia en general en el sector al por menor.

Las relaciones determinadas en el modelamiento se ratifican mutuamente en términos de covarianza y de varianza con estimadores similares $\left(0,98^{* * *}\right)$ que demuestran que los factores de apoyo directivo (FAD1 y FAD2) efectivamente hacen parte del constructo y efectivamente tienen efectos sobre consecuencia de carrera (Dawley, et al., 2010; Kwak, et al., 2010), dado que "consecuencia en la carrera" depende de las decisiones de apoyo directivo, asociados al apoyo brindado a los personales, independientemente de su género (Friedman y Greenhaus, 2001), permitiéndole un adecuado desarrollo profesional y familiar, confirmando que potenciar las medidas organizacionales de apoyo directivo inciden sobre el factor de consecuencias de carrera (Grandey y Cordeiro, 2002).

Se confirma además que las opciones organizacionales de conciliación analizadas en este trabajo como apoyo directivo (FAD1 y FAD2), demuestran efectos directos sobre la vida familiar (Machuca y Heredia, 2009), mejoran en general el clima organizacional (Bustamante et al., 2016) y evitan que los personales releguen dimensiones de la familia que producen bienestar, por una parte, en la medida que se reduzcan los efectos adversos sobre las consecuencias de carrera y por otra, se disminuyan las eventuales tendencias de conflicto trabajo-familia (Sivatte y Gauadamillas, 2014). Es así como los hallazgos del presente trabajo ratifican los planteamientos de diversos estudios confirmando que existe un efecto de la cultura trabajo-familia sobre el conflicto y el compromiso organizacional, similar a la relación que se produce entre el factor de consecuencia de carrera (FCC1), sobre satisfacción laboral (Thompson et. al, 1999; Allen, 2001) puesto que, efectivamente, ambientes de trabajo favorables incrementan el acceso a beneficios y fortalece la satisfacción laboral (Allen, 2001).

La hipótesis $\mathrm{H} 1$ que declara que apoyo directivo incide positivamente el ajuste trabajo familia a través de consecuencia de carrera (FCC1<-->FAP1, -0,414), define la preocupación de las jefaturas hacia las necesidades de los trabajadores (Thompson, et al., 1999) precisamente porque correlaciona negativamente con la interacción negativa trabajo-familia (FCC1<-->FAP2, -0,881), por una parte como señala Lapierre y Allen (2006) quienes confirmaron que políticas de flexibilidad con la familia inciden sobre la satisfacción personal y eventualmente también reduce conflictos de roles (Frye y Breaugh, 2004) y, por otra, porque en general el equilibrio familia-trabajo reduce en forma significativa las tensiones (Thompson, et al., 1999).

En general las personas independiente de su género declaran que lo más importante es el grupo familiar en sus vidas, porque la participación equitativa de hombres y mujeres (Allard, et al., 2011) proporciona a los empleados calidad de vida y porque existe la percepción que el equilibrio familia-trabajo (Casper, et al., 2011), sin embargo, en este trabajo se evidencian coincidencias de intereses y energías dedicadas al trabajo o a la familia, que conlleva observar cómo estas se afectan mutuamente y que diversos modelos de cultura trabajofamilia (Thompson, et al., 1999; Allen, 2001; Clark, 2001; McDonald, et al., 2005), confirman en la relación de los trabajadores con los supervisores quienes propician grados de flexibilidad operacional que incrementa el bienestar laboral (Casper y Harris, 2008). En consecuencia, efectivamente, prácticas laborales positivas pueden enriquecer la vida familiar y, por el contrario, si no existen los suficientes recursos y comienza aparecer el estrés, la relación es negativa y traerá consecuencias adversas en la conciliación trabajo - familia (Casper, et al., 2011), pudiendo generar conflicto en el contexto de desempeño (Machuca y Heredia, 2009). En este trabajo se ratifica, en consecuencia que las políticas de conciliación trabajo-familia (Thompson, et al.,1999) resultan positivamente valoradas por los empleados (Thompson et al., 2005) y además que, una gestión eficaz de apoyo directivo redunda en más y mejor desempeño con mínimo conflicto de roles (Clark, 2001).

En lo particular, se busca equilibrar los elementos de la vida familiar con las del trabajo (Kossek, et al., 2011) estableciendo una relación de conciliación trabajo-familia que disminuya el conflicto y mejore el bienestar de 
los empleados en atención a que el apoyo directivo resulta ser una dimensión vital de la gestión (Bustamante, et al. 2016) en tanto permite unificar el desempeño y valida el apoyo organizacional a los personales (Allen, 2001), primero, sobre la base de percepciones generales que, independientes del género, indican que las políticas de desarrollo de carrera (Casper, et al., 2011) resultan efectivamente apreciadas por hombres y mujeres sin distinción y, segundo, porque las políticas trabajo-familia propician la inclusión indiferente de los géneros (Machuca y Heredia, 2009) lo cual, por cierto, contribuye al desarrollo integral de la vida de los personales (Jansen, et al., 2010). Y, por lo mismo, una primera consecuencia, es que se incrementa el compromiso organizacional de los empleados, una segunda, se fortalece y retroalimenta la conciliación trabajo-familia (Thompson, et al., 1999), se incide en el bienestar organizacional (Lapierre y Allen, 2006) y, tercero, se ratifica la dimensión el apoyo directivo como mecanismo de gestión de la cultura trabajo-familia (Kossek, et al., 2011).

\section{CONCLUSIONES}

Se concluye que los principios de la cultura trabajo - familia confirman la incidencia apoyo directivo sobre consecuencias de carrera y fortalecen la confianza en la dirección, reducen las eventuales tendencias de conflicto y potencia la relación trabajo-familia y por consiguiente, incide en el bienestar organizacional. Se confirma la hipótesis que asume la incidencia de los factores de apoyo directivo sobre el factor consecuencias de carrera, en tanto inciden en las practicas organizacionales, conforme los principios que relacionan trabajo - familia, con lo que se reducen los efectos adversos sobre las consecuencias de carrera y se establece una relación de conciliación trabajo-familia que disminuye el conflicto y mejora el bienestar de los empleados. Por último, dado que el apoyo directivo incide positivamente el ajuste trabajo familia a través de consecuencia de carrera, se incrementa el compromiso organizacional de los empleados y se fortalece una percepción de bienestar que define una sensación de preocupación que puedan expresar las jefaturas hacia las necesidades de los trabajadores retroalimentando la conciliación trabajo- familia.

\section{REFERENCIAS}

Allard, K., Hass, L. y Philips, C., Family-Supportive Organizational Culture and Fathers' Experiences of Work-family Conflict in Sweden. https://doi.org/10.1111/j.1468-0432.2010.00540.x Gender, Work \& Organization. 18 (2), 141-157 (2011).

Allen, T. D., Family-Supportive Work Environments: The role of Organizational Perceptions. https://doi.org/10.1006/jvbe.2000.1774 Journal of Vocational Behavior, 58 (3), 414-435 (2001).

Bank, C., Kenny, B. y Stecher, A., Wal-Mart in the global South: Workplace culture, labor politics, and supply chains. Texas, TX: University of Texas Press. (2018).

Beauregard T. A. y Henry, L. C., Making the link Between Work-life Balance Practices and Organizational Performance. https://doi.org/10.1016/j.hrmr.2008.09.001b Human Resource Management Review, 19, pp. 9- 22. (2009).

Bustamante-Ubilla, M. A., Lapo, M. del C. y Grandón, M. L., Creación de un Cuestionario de Clima Organizacional para Hospitales de Alta Complejidad, Chile. https://doi.org/10.11144/Javeriana.rgyps15-30.ccco Revista Gerencia y Políticas de Salud. ISSN:1657-7027. 15 (30) 126 - 141 (2016).

Casper, W. J., Harris, C., Taylor-Bianco, A. y Holliday, J., Work-family Conflict, Perceived Supervisor Support and Organizational Commitment Among Brazilian Professionals. https://doi.org/10.1016/j.jvb.2011.04.011 Journal of Vocational Behavior, 79 (3), 640-652. (2011

Clark, S. C., Work Cultures and Work/family Balance. https://doi.org/10.1006/jvbe.2000.1759 Journal of Vocational Behavior, 58 (3), 348-365 (2001).

Dawley, D., Hougton, J. D. y Bucklew, N. S., Perceived Organizational Support and Turnover Intention: the Mediating Effects of Personal Sacrifice and Job Fit. https://doi.org/10.1080/00224540903365463 Journal of Social Psychology 150 (3), 238-257 (2010).

Debeljuh, P. y Jáuregui, K., Trabajo y Familia: Hacia una Cultura Familiar Amigable en el Contexto Latinoamericano. Esan, Cuadernos de Difusión 9 (16), 91-102. (2004).

Edgell, S., Gottfried, H. y Granter, E., The Sage Handbook of the Sociology of Work and Employment, (pp. 329- 347). London, UK: Sage. (2016).

Freiberg, A., Stover, J. B., De la Iglesia, G. y Fernández, M., Polychoric And Tetrachoric Correlations In Exploratory And Confirmatory Factorial Studies. Prensa Médica Latinoamericana - ISSN 1688-4094 Ciencias Psicológicas, VII (2): 151164 (2013).

Friedman, S. D. y Greenhaus, J. H., Work and Family—allies or Enemies? What Happens when Business Professionals Confront life Choices. New York: Oxford University Press. (2000).

Frye, N. K. y Breaugh, J. A., Family-friendly Policies, Supervisor Support, Work-Family Conflict, Family-Work Conflict, and satisfaction: A Test of a Conceptual Model. https://doi.org/10.1007/s10869-004-0548-4 Journal of Business and Psychology, 19 (2), 197-220 (2004). 
Durán, G. y Kremerman, M., Caracterización del Sector Retail - Comercio al por menor Una Mirada General, Fundación Sol, Cuadernos de investigación, N7 (2008).

Grandey, A. A. y Cordeiro, B. L., Family-Friendly Policies and Organizational Justice, a Sloan Work and Family Sloan Network Encyclopedia Entry (2002).

Hernández, S. R., Fernández, C. y Baptista, P., Metodología de la Investigación. Cuarta Edición. Editorial Mc Graw Hill Interamericana pág. 839 (2006).

Jansen, N., Mohren, D., Van Amelsvoort, L., Janssen, N. y Kant, I., Changes in Working Time Arrangements Over Time as a Consequence of Work-Family Conflict. https://doi.org/10.3109/07420528.2010.489874 Chronobiology International, 27(5), 1045-1061 (2010).

Kossek, E. E., Baltes, B. B. y Matthews, R. A., How Work-Family Research Can Finally Have an Impact in Organizations. https://doi.org/10.1111/j.1754-9434.2011.01353.x Industrial \& Organizational Psychology. 4 (3), $352-369$ (2011).

Kwak, C., Chung, B. Y., Xu, Y. y Eun-Jung, Ch., Relationship of Job Satisfaction with Perceived Organizational Support and Quality of Care Among South Korean nurses: A questionnaire Survey. https://doi.org/10.1016/j.ijnurstu.2010.02.014 International Journal of Nursing Studies, 47(10), 1292-1298 (2010).

Lapierre, L. M. y Allen, T. D., Work-Supportive Family, Family.-Supportive Supervision, use of Organizational Benefits, and Problem-Focused Coping: Implications for Work-Family Conflict and Employee Well-Being. https://doi.org/10.1037/10768998.11.2.169 Journal of Occupational Health Psychology, 11(2), 169-181 (2006).

Lewis, S. y Smithson, J., Sense of Entitlement to Support for the Reconciliation of Employment and Framily Life. https://doi.org/10.1177/00187267015411003 Human Relations, 54(11), 1455-1481 (2001)

Litchfield, L. C., Swanberg, J. E. y Sigworth, C. M., Increasing the Visibility of the Invisible Workforce: Model Programs and Policies for Hourly and Lower Wage Employees. Final Report; Boston: Boston College Center for Work \& Family, Carroll School of Management April, (2004).

Jáuregui, M. K. y Bejarano, H. A., Apoyo para Desarrollo de Carrera de Miembros de "Familias de Doble Carrera. Revista Venezolana de Gerencia, 14(48), 507-517. (2009).

Mauno, S., Kinnunen, U. y Pyykkö, M., Does Work-Family Conflict Mediate the Relationship Between Work-Family Culture and Self Reported Distress? Evidence for five Finnish Organizations. https://doi.org/10.1348/096317905X37082 Journal of Occupational and Organizational Psychology, 78(4), 509-530 (2005).

Shaffer, M. A., Joplin, J. y Yu-Shan, H., Expanding the Boundaries of Work-family. A review and Agenda for Future Research. https://doi.org/10.1177/147059581139880 International Journal of Cross Cultural Management, 11(2), 221268. (2011).

De Sivatte, I. y Guadamillas, F., ¿La Disponibilidad de Medidas de Conciliación y la Cultura Trabajo Familia Disminuyen el Conflicto Trabajo-Familia de los Empleados?_Universia Business Review, 44, 54-71 (2014).

Spielberger, C., Reheiser, E. C., Reheiser, J. E. y Vagg, P. R., Measuring Stress in the Workplace: The Job Stress Survey. Stress and Health; Research and Clinical Applications, Amsterdam, The Netherlands: Harwood Academic Publishers. 397409. (2000).

Thompson, C. A., Beauvais, L. L. y Lyness, K. S., When Work-Family Benefits are not Enough: The influence of WorkFamily Culture on Benefit Utilization, Organizational Attachment, and Work-Family Conflict. https://doi.org/10.1006/jvbe.1998.1681 Journal of Vocational Behavior, 54(3), 392-415 (1999).

Tilly, C. y Carré, F., Endnote: Retail Work - Perceptions and Reality. En: Irena Grugulis y Odul Bozkurt, Retail work, (pp. 297-306). Basingstoke, UK: Palgrave. (2011).

Warhurst, C. y Nickson, D., Employee Experience of Aesthetic Labour in Retail and Hospitality. https://doi.org/10.1177/0950017007073622 Work, Employment and Society, 21(1), 103-120 (2007). 